\title{
Entwicklung therapeutischer Peptidimpfstoffe gegen Krebs
}

\author{
H.-G. Rammensee ${ }^{1}$ \\ ${ }^{1}$ Direktor des Instituts für Zellbiologie, Universität Tübingen, \\ Tübingen
}

Krebszellen unterscheiden sich immer von normalen Körperzellen. Die Unterschiede sind einerseits bedingt durch die genetischen Veränderungen, die zur Entartung geführt haben, etwa Mutationen oder Translokationen, die die Regulation der Zellteilung, der Kontaktinhibition oder der Apoptoseresistenz beeinflussen, andererseits durch Konsequenzen dieser Veränderungen wie zum Beispiel die Reexpression von embryonalen Genen, oder die Überexpression von ansonsten normalen Genen, oder die Expression von viralen Genen bei virusinduzierten Tumoren. Alle diese Veränderungen können prinzipiell vom Immunsystem erkannt werden, was zur Abtötung oder anderweitiger Beeinflussung der sie tragenden Zellen führen kann. Zusätzlich zu diesen Krebsantigenen kommen für eine Immuntherapie auch gewebe- oder zelltypspezifische Strukturen infrage, solange der Tumor von einem nicht lebensnotwendigen Zelltyp ausgeht.

Wir teilen die für eine Immuntherapie geeigneten Antigene in 3 Kategorien ein (Abb. 1).

1. Kategorie: Tumorspezifische Antigene, die nur auf Tumorzellen und sonst nirgends und zu keiner Zeit im Körper vorkommen. Dieses sind ausschließlich durch Mutationen in den Krebszellen oder von Tumorviren hervorgerufene Strukturen. Mutationen gibt es viele in jeder Tumorzelle, darunter solche, die für das maligne Verhalten ursächlich sind („Driver-Mutationen“) und weitere, die für die Tumorzellen eher belanglos sind („Passenger-Mutationen“). Mehrere hundert Mutationen sind regelmäßig in Tumorzellen zu finden. Allerdings ist das Spektrum der Mutationen in jedem Tumor, von Patient zu Patient, fast völlig unterschiedlich.

2. Kategorie: tumorassoziierte Antigene. Dazu gehören reexprimierte embryonale Antigene („Cancer testis antigens“, Beispiel NY-ESO1) sowie in der Tumorzelle überexprimierte, also in höherer Molekülzahl als in normalen Zellen vorhandene, ansonsten aber normale zelluläre Strukturen (Beispiel HER2-neu). Hierzu gehören auch durch posttranslationale Modifikationen besonders in Tumorzellen hervorgerufene Veränderungen.

3. Kategorie: Differenzierungsantigene. Diese sind die bereits erwähnten zelltypspezifischen Strukturen, die nur für Tumorentitäten mit Urspung aus nicht überlebensnotwendigen Geweben relevant sind. Man nimmt mit einer gegen Differenzierungsantigene gerichteten Immuntherapie daher bewusst in Kauf, dass auch die gesunden Zellen des betreffenden Gewebes zerstört werden. Beispiel aus der passiven, antikörpervermittelten Immuntherapie: CD20, eine Struktur auf normalen B-Zellen, aber auch auf B-Zelllymphomen.

Alle diese Antigene können vom adaptiven Immunsystem erkannt werden, also von Antikörpern oder von T-Zellen. Allerdings sind in diesem Zusammenhang für Antikörper nur diejenigen Antigene relevant, die auf der Zelloberfläche direkt 


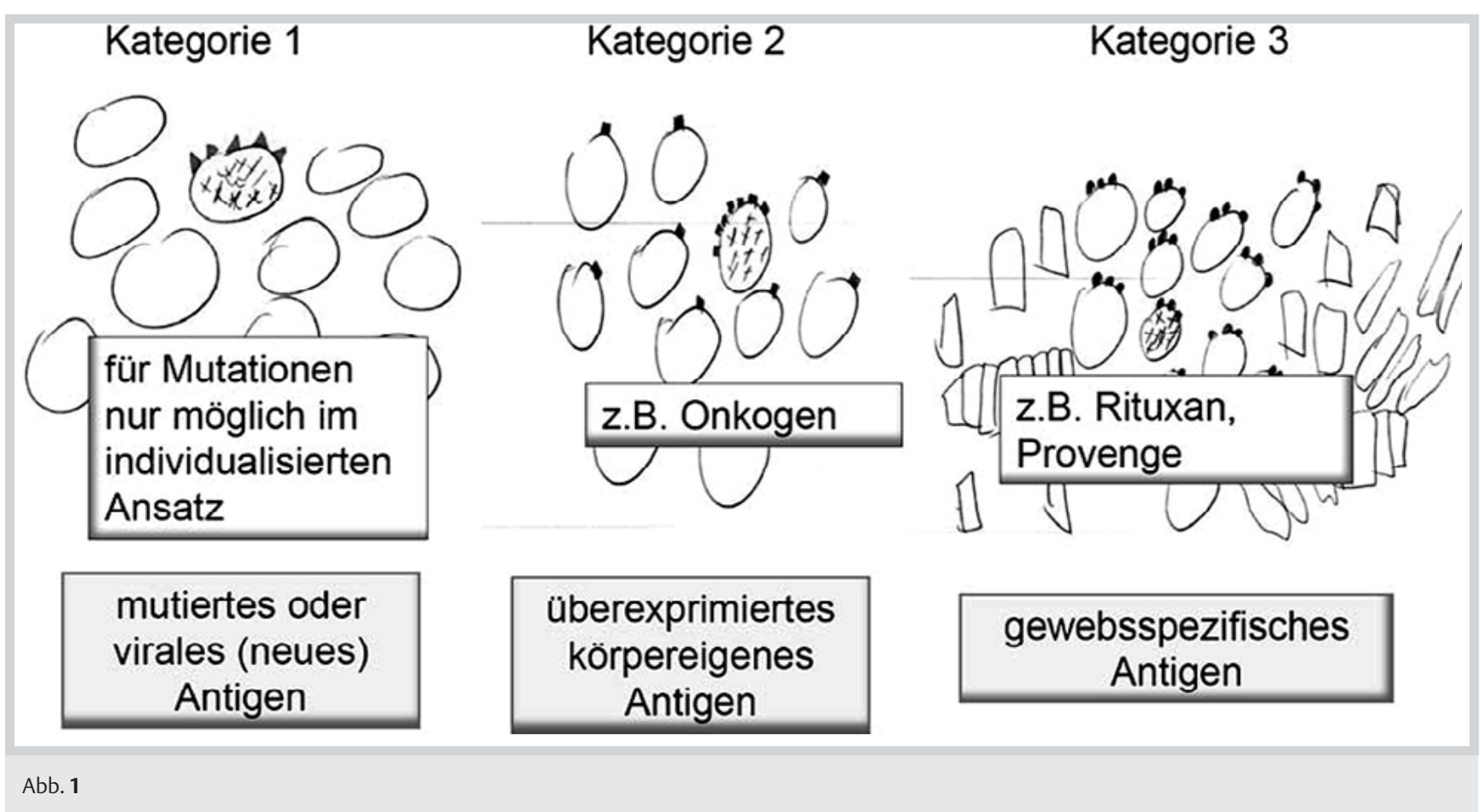

exprimiert sind. Die Auswahl solcher Strukturen ist auf relativ wenige begrenzt, und aus praktischen Gründen kommen antikörpervermittelte Immuntherapien, die insbesondere passive Immunisierungen sind, fast nur für Antigene der Kategorien 2 und 3 in Betracht. Dagegen sind T-Zellen prinzipiell in der Lage, Fragmente von jedwedem zellulären Protein, wo immer es in der Zelle auch vorkommt, auf der Zelloberfläche als HLA-präsentiertes Peptid zu erkennen.

Ziel der therapeutischen Impfung ist es, geeignete Tumorantigene so zu applizieren, dass sie eine effiziente Immunantwort gegen die Krebszellen hervorrufen. Dabei ist zu berücksichtigen, dass diese Antigene bereits auf den Tumorzellen vorhanden sind, jedoch in der Regel noch nicht zu einer Erstaktivierung der spezifischen Immunzellen geführt haben. Dies deshalb, weil einerseits die für eine erstmalige Aktivierung von T-Zellen erforderlichen Kostimulationssignale, wie die Interaktion von CD80/86 auf dendritischen Zellen mit CD28 auf T-Zellen, im Tumormilieu nicht vorhanden sind, und andererseits im Tumor immunsuppressive Bedingungen herrschen. Man versucht deshalb bei einer therapeutischen Vakzinierung, durch Applikation der Tumorantigene fern des Tumors, in der Regel intradermal oder subkutan, zusammen mit einem Adjuvans, die T-Zellen zu aktivieren und hofft, dass diese dann zu den Tumorzellen migrieren, wobei man am besten auch gleich versucht, das Tumormilieu so zu beinflussen, dass eine Immunantwort dort nicht gleich unterdrückt wird.

Die Identifizierung der Tumorantigene kann mittels einer Reihe unterschiedlicher Strategien erfolgen. Die ersten Tumorantigene wurden durch Spezifitätsanalysen von T-Zellen oder Antkörpern identifiziert, die spontan in Patienten aufgetreten waren [1,2]. Später wurden Antigene und darin TZellepitope identifiziert, die von Genprodukten stammen, die als tumorassoziiert gelten. Wir identifizieren direkt die relevanten Antigene, die in Form von Peptiden auf den HLA-
Molekülen auf Tumorzellen präsentiert werden, und verwenden als Quelle hierfür frisch exstirpiertes Tumorgewebe aus Patienten $[3,4]$. Bei diesem Verfahren werden die HLA-Moleküle des Tumors sowie des relevanten Normalgewebes, sofern vorhanden, immunpräzipitiert, die gebundenen Peptide werden eluiert, mittels HPLC aufgetrennt und mit hochauflösender Massenspektrometrie identifiziert. Ziel ist es, solche HLA-Liganden, also Peptide, zu finden, die im Tumor vorhanden sind, im Normalgewebe jedoch nicht, oder zumindest im Tumor in höherer Kopienzahl gegenüber normalen Zellen vorkommen.

Die Form des Antigens für die Impfung kann sehr unterschiedlich gewählt werden. Die Minimalform ist ein synthetisches Peptid, das genau dem natürlichen Peptid auf der Tumorzelle entspricht [5]. Das Peptid kann auch verlängert werden; es muss dann nach der Impfung erst von antigenpräsentierenden Zellen, insbesondere dendritischen Zellen, aufgenommen und prozessiert werden [6]. Man kann auch antigenkodierende DNA oder RNA einsetzen, wobei RNA besonders vielversprechend ist, da die RNA keine Komplikationen durch unerwünschte Insertion ins Genom verursachen und außerdem selbst als Adjuvans wirken kann [7]. Die erste von der FDA zugelassene therapeutische Krebsimpfung ist ein rekombinantes Protein, das mit kostimulierenden Molekülen versehen ist [8]. Auch virale oder bakterielle Konstrukte, in die Gene für Tumorantigene inseriert sind, werden erprobt. Wir entwickeln die Tumor-Vakzinierung mit den natürlichen Peptiden genau entsprechenden synthetischen Peptiden. Dabei muss man zunächst die Einschränkung in Kauf nehmen, dass solche Peptide immer genau zu den HLA-Molekülen des Patienten passen müssen; in den bisherigen Studien waren dies meist HLA-A2-exprimierende Patienten. In Mitteleuropa tragen knapp die Hälfte der Bevölkerung dieses Merkmal. Aus der Analyse von hunderten von Nierenzellkarzinomproben wurden 10 solcher Peptide ausgewählt, die häufig auf HLA- 
A2-Molekülen als tumorassoziierte Peptide zu finden waren, z.B. aus dem c-met-Protoonkogen oder aus Zellzyklus oder Zelltod regulierenden Genprodukten. Eine ausführliche Darstellung einer klinischen Phase-I- und einer darauf aufbauenden Phase-II-Studie mit zusammen 96 Patienten wurde kürzlich veröffentlicht [5,9]. Die Vakzinierung erfolgte subkutan mit dem Zytokin GM-CSF als Adjuvans und bei einem Teil der Patienten mit einem Immunmodulator, der die Immunsuppression reduzieren sollte. Es zeigte sich eine eindrucksvolle Verlängerung des Überlebens der Patienten, die eine starke Immunantwort gegen den Peptid-Cocktail zeigten, wobei die Nebenwirkungen sehr viel geringer waren als bei Standardtherapien mit Tyrosinkinaseinhibitoren, sowie aufschlussreiche Korrelationen mit Biomarkern, die folgende Studien verbessern können sollten. Wie auch von anderen bisher durchgeführten therapeutischen Tumor-Vakzinierungs-Studien bekannt, zeigte die Impfung jedoch keinen bedeutenden Einfluss auf die Tumorprogression. Es besteht daher noch wesentlicher Optimierungsbedarf, der durch die bisherigen Erfahrungen mit der erwähnten und mit weiteren Studien auch anderer Strategien aussichtsreich bearbeitet werden kann.

Wir verfolgen derzeit 2 unterschiedliche Strategien, um die Immuntherapie mit Peptiden weiterzuentwickeln. Ein Ziel ist die Zusammenstellung von Peptid-Cocktails mit 20-30 Peptiden, die für häufige HLA-Moleküle passen, also neben HLA-A2 etwa auch für HLA-A1, B7 oder B8, um alle Patienten ohne vorherige HLA-Typisierung behandeln zu können. Dies wird derzeit für das Prostatakarzinom bei prostatektomierten Patienten vorbereitet, wobei in diesem Fall sowohl tumorassoziierte (Kategorie 2) als auch prostatagewebsspezifische (Kategorie 3) zum Einsatz kommen sollen [10]. Ein weiteres Ziel ist die Verwendung von wirklich tumorspezifischen Peptiden (Kategorie 1), die eine wesentlich stärkere Immunantwort hervorrufen sollten, da gegen diese keine zentrale, also bereits im Thymus festgelegte, Toleranz des Immunsystems vorliegt. Hierbei kommt man jedoch nicht umhin, vollkommen patientenindividuell vorzugehen, da nicht nur die tumorspezifischen Mutationen in jedem Patienten andere sind, sondern auch die HLA-Moleküle [11]. Wir arbeiten daher derzeit an der Entwicklung der geeigneten Logistik für ein solches Vorgehen. Neben der Identifizierung der geeigneten mutationstragenden Peptide durch Kombination von Genom- und Transkriptom-Sequenzierung einerseits und massenspektrometrischer Peptidanalyse andererseits, jeweils aus Tumorproben des individuellen Patienten, müssen die zur Impfung vorgesehenen Peptide nach dem Arzneimittelgesetz hergestellt werden. Hierfür wurde an der Universität Tübingen mit großem Aufwand die notwendige Infrastruktur geschaffen.

Wir erwarten in den nächsten Jahren eine günstige Entwicklung der aktiven spezifischen Immuntherapie [12], wobei die bisherigen Erkenntnisse Lösungen der erkannten Probleme erleichtern. Danach sollte eine erfolgreiche Immuntherapie nach chirurgischer Resektion mehrere möglichst spezifische Antigene zusammen mit geeigneten Adjuvanzien und Immunmodulatoren einsetzen und die lokale Immunsuppression im Tumorbereich zusätzlich durch Kombination mit geeigneter Chemo/Radiotherapie beeinflusst werden.
Interessenkonflikt: Der Autor ist beteiligt an den Firmen immatics, CureVac, Bamomab und Synimmune, Antragsteller bei Drittmittelprojekten von u.a. DFG, BMBF, EU. Er ist oder war Berater bei verschiedenen Konsortien und Pharmafirmen, ist Mitinhaber von Patenten im Bereich der Immuntherapie und hält sporadisch auch vergütete Vorträge.

\section{Literatur}

1 van der Bruggen P, Traversari C, Chomez P et al. A gene encoding an antigen recognized by cytolytic T lymphocytes on a human melanoma. Science $1991 ; 254: 1643-1647$

2 Türeci O, Sahin U, Pfreundschuh M. Serological analysis of human tumor antigens: molecular definition and implications. Mol Med Today 1997; 3: $342-349$

3 Falk K, Rötzschke O, Stevanović $S$ et al. Allele-specific motifs revealed by sequencing of self-peptides eluted from MHC molecules. Nature 1991; 351: $290-296$

4 Singh-Jasuja H, Emmerich NP, Rammensee HG. The Tübingen approach: identification, selection, and validation of tumor-associated HLA peptides for cancer therapy. Cancer Immunol Immunother 2004; 53: $187-195$

5 Weinschenk T, Gouttefangeas C, Schirle M et al. Integrated functional genomics approach for the design of patient-individual antitumor vaccines. Cancer Res 2002; 62: 5818-5827

6 de Vos van Steenwijk PJ, Ramwadhdoebe TH, Löwik MJ et al. A placebocontrolled randomized HPV16 synthetic long-peptide vaccination study in women with high-grade cervical squamous intraepithelial lesions. Cancer Immunol Immunother 2012; 61: 1485-1492

7 Weide B, Pascolo S, Scheel B et al. Direct injection of protamine-protected mRNA: results of a phase $1 / 2$ vaccination trial in metastatic melanoma patients. J Immunother 2009; 32: 498-507

8 Kantoff PW, Higano CS, Shore ND et al.; IMPACT Study Investigators. Sipuleucel-T immunotherapy for castration-resistant prostate cancer. N Engl J Med 2010; 363: 411-422

9 Walter S, Weinschenk T, Stenzl A et al. Multipeptide immune response to cancer vaccine IMA901 after single-dose cyclophosphamide associates with longer patient survival. Nat Med 2012 Jul 29 [Epub ahead of print]

10 Feyerabend S, Stevanovic S, Gouttefangeas C et al. Novel multi-peptide vaccination in Hla-A2+ hormone sensitive patients with biochemical relapse of prostate cancer. Prostate 2009; 69: 917-927

11 Rammensee HG, Weinschenk T, Gouttefangeas $C$ et al. Towards patientspecific tumor antigen selection for vaccination. Immunol Rev 2002; 188: $164-176$

12 Topalian SL, Weiner GJ, Pardoll DM. Cancer immunotherapy comes of age. J Clin Oncol 2011; 29: 4828-4836

Bibliografie

DOI http://dx.doi.org/10.1055/s-0032-1324901

Arzneimittelforschung 2012; 62, Suppl. 1: S5-S7

(c) Georg Thieme Verlag KG Stuttgart · New York .

ISSN 0004-4172

Please note that the print version was published with a different, incorrect DOI. The correct DOI is the one shown here. 